\title{
Molecular Motion of Polyethylene Oxide in the Urea Complex
}

\author{
Kunio Hikichi, ${ }^{\dagger}$ Daisuke Oka, Toshiyuki Shibata, ${ }^{*}$ \\ and Jun Uzawa*
Department of Polymer Sciences, Faculty of Science, Hokkaido University, Sapporo 060-0810, Japan
* Biopolymer Physics Laboratory and Division of Molecular Characterization, The Institute of Physical and Chemical Research, 2-1 Hirosawa, Wako 351-0198, Japan

(Received March 31, 1999)

\author{
KEY WORDS Polyethylene Oxide Urea Complex/Molecular Motion ${ }^{13} \mathrm{C}$ Nuclear Magnetic \\ Resonance ${ }^{1} \mathrm{H}$ Nuclear Magnetic Resonance
}

It has been known for many years that polyethylene oxide (PEO) forms an inclusion complex with urea. ${ }^{1}$ The PEO chain is accommodated in the channel formed by urea. Bulk PEO is in a 7-residue 2-turn helix with tgt conformation in the crystalline state. It has been reported that PEO-urea complex formed from solution has a trigonal crystal structure, while recrystallization from the melt produces a hexagonal form. The conformation in the PEO-urea complex is more extended than that of bulk PEO. ${ }^{2,3}$

Many years ago we reported that the chain of PEO in the crystalline phase starts to undergo cooperative rotational oscillation about the helical axis at $270 \mathrm{~K} .{ }^{4}$ Tonelli and co-workers investigated motions of PEO in the inclusion complex with urea by the ${ }^{13} \mathrm{C}$ relaxation. ${ }^{2}$ They reported a significant retardation of $\mathrm{PEO}$ chain motion in the complex compared to polytetrahydrofuran (PTHF) in the urea complex, in which PTHF chain has much greater mobility than in bulk PTHF. ${ }^{5}$

The purpose of this paper is to undertake more detail study on motional behavior of PEO chain in the urea complex using the solid state ${ }^{13} \mathrm{C}$ NMR and broad-line ${ }^{1} \mathrm{H}$ NMR methods.

\section{EXPERIMENTAL}

\section{Sample}

Polyethylene oxide of nominal molecular weight of 6000 was obtained from Aldrich Chemical Co. The complex was obtained by adding PEO to saturated methyl alcohol solution of deuterated urea. The white powder of complex was precipitated; it was dried under a reduced pressure at $313 \mathrm{~K}$ for a week.

\section{NMR Measurement}

Solid-state ${ }^{13} \mathrm{C}$ NMR measurements at a frequency of $68 \mathrm{MHz}$ were performed using a JEOL JNM-GX270 spectrometer in a mode of magic-angle rotation ( $c a$. $5.5 \mathrm{kHz})$ and high-power proton decoupling $(55 \mathrm{kHz})$. Proton irradiation frequency was set $3 \mathrm{ppm}$ downfield from tetramethylsilane (TMS). The chemical shift was measured from methylene carbon of adamantane (29.5 $\mathrm{ppm})$. Otherwise noted, the cross-polarization (CP)

$\dagger$ To whom all correspondence should be addressed. was used to enhance the signal. Temperature was calibrated using methyl alcohol. ${ }^{13} \mathrm{C}$ spin-lattice relaxation time $T_{1 \mathrm{C}}$ and ${ }^{1} \mathrm{H}$ spin-lattice relaxation time in rotating frame $T_{1 \rho \mathrm{H}}$ were measured by the Torchia pulse sequence ${ }^{6}$ and the spin-locking method, ${ }^{7}$ respectively.

The continuous wave $(\mathrm{CW}){ }^{1} \mathrm{H}$ NMR spectra were measured as a function of temperature at a frequency of $22 \mathrm{MHz}$ using a home-made spectrometer with a Robinson type detector. The second moment of resonance line was calculated from the observed derivative curve of absorption line.

In order to ensure the trigonal crystal form we did not make measurements for samples which had been once melt.

\section{RESULTS AND DISCUSSION}

Figure 1 shows ${ }^{13} \mathrm{C}$ spectra of pure urea (a), PEO-urea complex (b), and pure PEO (c) at room temperature. Spectrum (a) was observed without CP, (b) was observed at a CP contact time of $0.5 \mathrm{~ms}$, and (c) at $0.1 \mathrm{~ms}$. Signals appearing at $125 \mathrm{ppm}$ in spectra (a) and (c) arise from the sample holder. A broad signal seems to appear in spectrum (a) between 40 to $100 \mathrm{ppm}$. This is probably due to the background distortion caused by the low sensitivity of deuterated urea. A peak appearing at about $163 \mathrm{ppm}$ in spectrum (a) is assigned to carbonyl carbon of urea, which is slightly shifted to $164 \mathrm{ppm}$ in spectrum (b). The peak maximum of methylene carbon of PEO appears at $72 \mathrm{ppm}$ for pure PEO and $70.6 \mathrm{ppm}$ for the complex. It is of interest to note that the signal of complex is sharper than that of pure PEO.

Table I shows $T_{1 \mathrm{C}}$ and $T_{1 \rho \mathrm{H}}$ for pure PEO and PEO-urea complex at room temperature. $T_{1 \mathrm{C}}$ has two components, longer and shorter, for both samples. The longer component has been attributed to crystalline phase and the shorter one to amorphous phase. ${ }^{2}$ When PEO forms complex with urea, the longer component becomes shorter, while the shorter one remains unchanged. The differences in $T_{1 \mathrm{C}}$ between pure PEO and the complex observed in this work are mostly consistent with those reported previously. ${ }^{2} T_{1 \rho \mathrm{H}}$ is greater for the complex than for pure PEO.

Figure 2 shows the line-width of methylene carbon signal of PEO as a function of temperature for pure PEO 


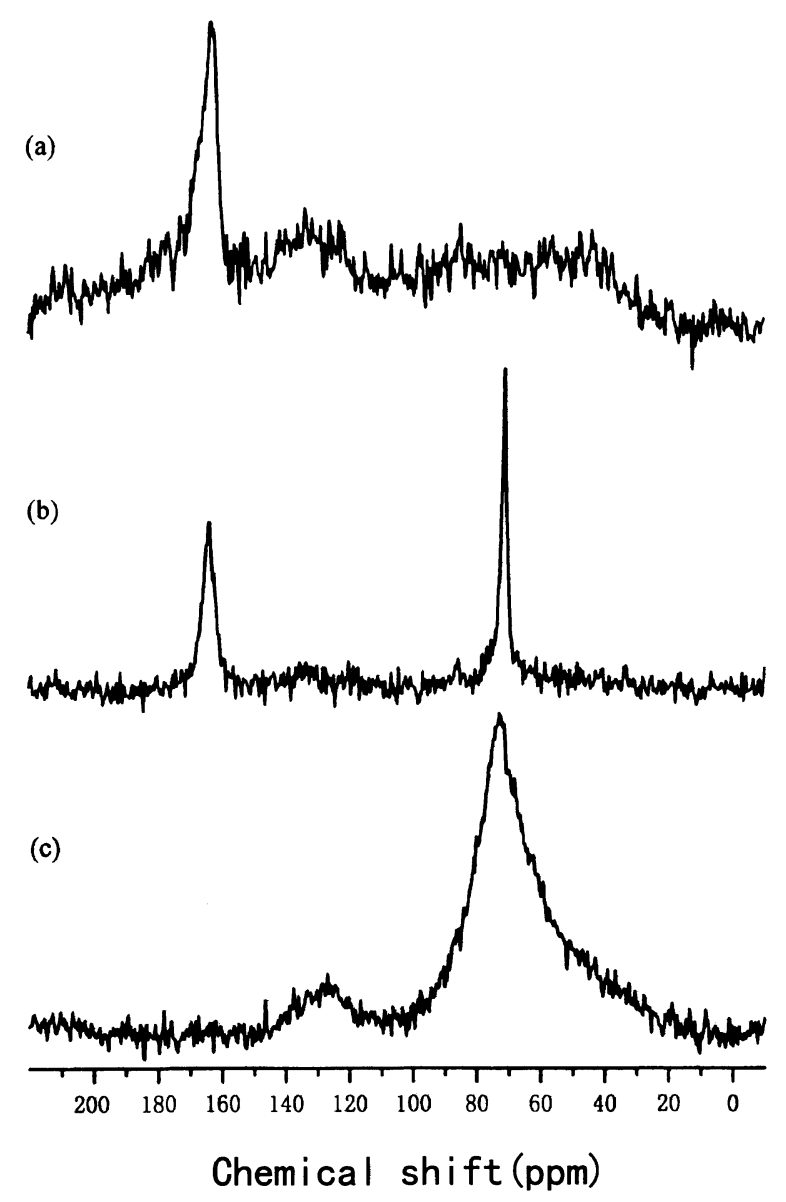

Figure 1. ${ }^{13} \mathrm{C}$ spectra of pure urea (a), PEO-urea complex (b), and pure $\mathrm{PEO}(\mathrm{c})$ at room temperature. Spectrum (a) was observed without CP. (b) was observed at a CP contact time of $0.5 \mathrm{~ms}$, and (c) at $0.1 \mathrm{~ms}$. A peak at $125 \mathrm{ppm}$ arises from the sample holder.

Table I. ${ }^{13} \mathrm{C}$ spin-lattice relaxation time $T_{1 \mathrm{C}}$ and ${ }^{1} \mathrm{H}$ spin-lattice relaxation time in rotating frame $T_{1 \rho \mathrm{H}}$ for pure PEO and PEO-urea complex at room temperature

\begin{tabular}{llc}
\hline Sample & $T_{1 \mathrm{c}} / \mathrm{s}$ & $T_{1 \rho \mathrm{H}} / \mathrm{ms}$ \\
\hline Pure PEO & 9 & 0.2 \\
& 0.3 & \\
\hline PEO-urea complex & 2 & 2 \\
& 0.4 & \\
\hline
\end{tabular}

and PEO-urea complex. We find that the temperature dependence of line-width shows a maximum at $310 \mathrm{~K}$ for pure $\mathrm{PEO}$, but it is a horizontal straight line for the complex. Since such a maximum is caused by the reduced decoupling effect due to an interference between molecular motion and proton decoupling $(55 \mathrm{kHz}),{ }^{8,9}$ the chain in bulk PEO undergoes motion at a frequency of $55 \mathrm{kHz}$ at a temperature of maximum $(310 \mathrm{~K})$. This is in agreement with our previous conclusion. ${ }^{4}$ PEO chain undergoes a remarkable motion in the vicinity of room temperature.

On the other hand, the line-width of PEO in the complex remains unchanged with varying temperature. It means that at all temperatures studied molecular motion is not in the range of $55 \mathrm{kHz}$.

The correlation time of motion of pure PEO below

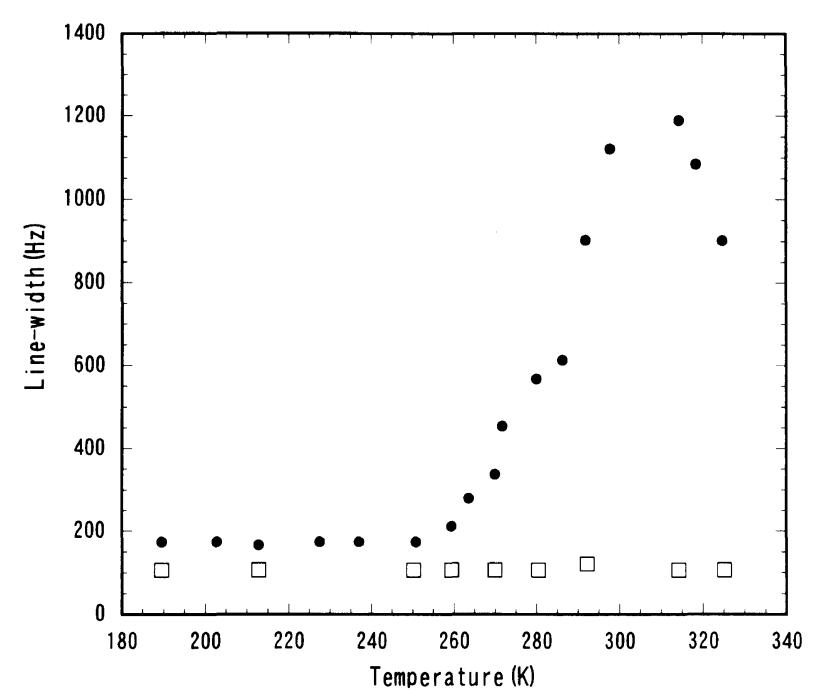

Figure 2. The line-width of methylene carbon signal as a function of temperature for pure PEO (O) and PEO-urea complex ( $\square$ )

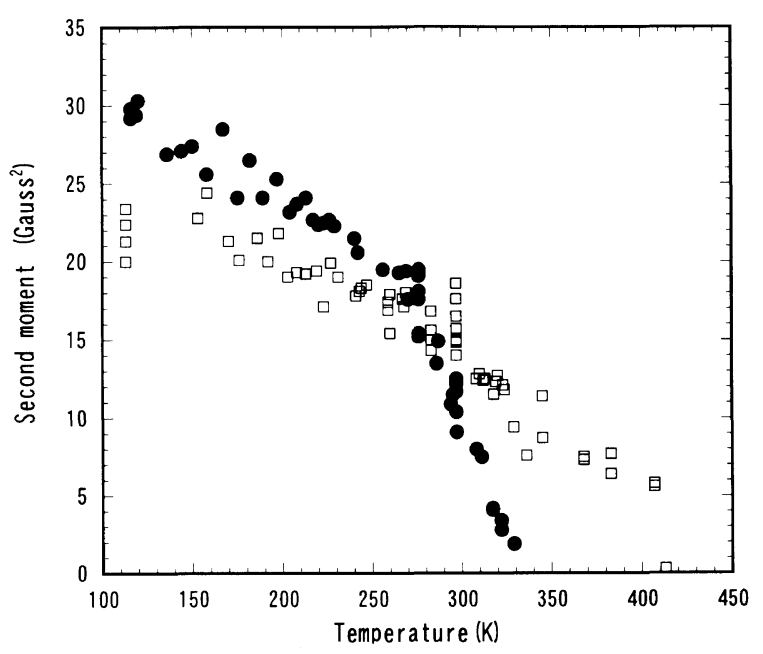

Figure 3. The second moment of ${ }^{1} \mathrm{H}$ NMR spectra as a function of temperature for pure PEO (O) and PEO-urea complex ( $\square$ ).

$310 \mathrm{~K}$ is greater than the inverse of $55 \mathrm{kHz}$ (greater than the inverse of carbon frequency). If we simply explain the reduction in $T_{1 \mathrm{C}}$ from $9 \mathrm{~s}$ of bulk to $2 \mathrm{~s}$ of the complex as a variation of motional state, it implies that the motion is faster for the complex than for pure PEO. However, the results of broad-line ${ }^{1} \mathrm{H}$ spectra give opposite results.

Figure 3 shows the second moment of ${ }^{1} \mathrm{H}$ NMR spectra of pure PEO and PEO-urea complex as a function of temperature. At lower temperatures the PEO-urea complex shows lower second moment values than pure PEO. This is due to the reduced intermolecular interaction between PEO chains in the PEO-urea complex. Pure PEO shows a remarkable decrease in second moment above a temperature of $280 \mathrm{~K}$. This is in agreement with our previous results, ${ }^{4}$ which suggested the cooperative rotational oscillation of PEO in the bulk. On the other hand, PEO in the urea complex shows a rather gradual decrease up to a temperature of $410 \mathrm{~K}$, where the complex melts.

The results indicate that the motion of PEO chain in the urea complex is much hindered as compared to pure PEO. The chain of PEO in the complex does not show such a cooperative motion as that of pure PEO; the rate 
of motion for the complex is less than $55 \mathrm{kHz}$ at all temperatures studied. This is in contrast to the cases of PTHF urea complex ${ }^{5}$ and polyethylene urea complex, ${ }^{10}$ in which the polymer chains in complex become more mobile than pure polymers. A reason of this contrast is probably the formation of hydrogen bond between oxygen atom of PEO and urea as suggested by Tonelli et al. ${ }^{2,3}$

It is rather difficult to explain the variation of $T_{1 \mathrm{C}}$ from pure PEO to the complex. The increase in $T_{1 \rho \mathrm{H}}$ from $0.2 \mathrm{~ms}$ of bulk to $2 \mathrm{~ms}$ of the complex may be caused by the reduced intermolecular interaction for the complex. We need further study on the relaxation times as function of temperature.

Acknowledgment. This work was supported by the special Grant-in-Aid for Promotion of Education and Science in Hokkaido University provided by the Ministry of Education, Science, Sports, and Culture of Japan.

\section{REFERENCES}

1. H. Tadokoro, T. Yoshihara, Y. Chatani, and S. Murahashi, J. Polym. Sci. B, 2, 363 (1964).

2. N. Vasanthan, I. D. Shin, and A. E. Tonelli, Macromolecules, 29, 263 (1996).

3. A. Chenite and F. Brisse, Macromolecules, 24, 2221 (1991).

4. K. Hikichi and J. Furuichi, J. Polym. Sci., Polym. Phys. Ed., 6 653 (1968).

5. N. Vasanthan, I. D. Shin, and A. E. Tonelli, J. Polym. Sci. B, 33, 1385 (1995).

6. D. A. Torchia, J. Magn. Reson., 30, 613 (1978).

7. D. E. Axelson and K. E. Russell, Prog. Polym. Sci., 11, 221 (1985).

8. K. Takegoshi and K. Hikichi, J. Chem. Phys., 94, 3200 (1991).

9. D. Suwelack, W. P. Rothwell, and J. S. Waugh, J. Chem. Phys., 73, 2559 (1980).

10. P. Sozzani, F. A. Bovey, and F. C. Shilling, Macromolecules, 24, 6764 (1991). 